\title{
Research Paper \\ Adjustment of Optimal Sports Site Selection Criteria for Elderly Using Analytical Hierarchy Process and Geographic Information System
}

\author{
*Omid Jamshidi ${ }^{1}$, Morteza Doostipasha ${ }^{1}$, Seyed Mohamad Hosein Razavi ${ }^{1}$, Mahmood Gudarzi ${ }^{1}$
}

1. Department of Sports Management, Faculty of Physical Education and Sports Sciences, University of Mazandaran, Babolsar, Iran.

Received: 18 Jun. 2017 Accepted: 25 Sep. 2017

Key words:

Adjustment, Sports Facilities, Elderly
Cittation: Jamshidi O, Doostipasha M, Razavi SMH, Gudarzi M. [Adjustment of Optimal Sports Site Selection Criteria for Elderly Using Analytical Hierarchy Process and Geographic Information System (Persian)]. Iranian Journal of Ageing. 2018; 12(4):506-517. https://doi.org/10.21859/SIJA.12.4.506

d ofi": https://doi.org/10.21859/SIJA.12.4.506

\begin{abstract}
A B STRACT
Objectives Aging and its associated problems are one of the challenges in the country. This necessitates the need for proper planning during this period. In this regard, the present study was conducted with the aim of fitting the indicators for choosing optimal sports location for the elderly.

Methods \& Materials The present study is a descriptive-analytic research that determined the weights of research criteria using a structured interview with eight experts in Analytical Hierarchy Process. In addition, the pools of Karaj (27 cases) were selected as the study population and the elderly people referred to these pools were selected as the research community. A total of 127 elderly were examined using stratified sampling. After weighing the sub-criteria of each research criterion, AutoCAD, Excel, ArcGIS, and Google Earth software were used to create a spatial database.

Results Of the total 127 elder participants, $66 \%$ were male, $48 \%$ were aged $60-65$ years, and $54 \%$ spent more than 6 hours a week on sports. According to experts' opinions, three indicators of accessibility, proximity to other land uses, and safety ranked higher in the field of influencing the selection of sports places. Among the sub-criteria, subway lines and highways for access index; proximity to parks and green spaces and proximity to cultural centers for proximity index; and proper position to health centers and fire centers for safety index had relative importance compared to other sub-criteria.

Conclusion Proximity to other land uses and safety was recognized as important criteria influencing the selection of sports places. By using the results of this research and by correctly locating the sports venues, it is possible to prevent the mistakes in choosing the location of sports facilities.
\end{abstract}

\section{Extended Abstract}

\section{Objectives}

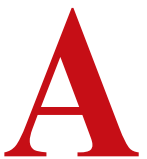

ging is a critical period of life and considering the issues and needs of this stage is a social necessity. It should be noted that lack of attention to health and neglecting the behaviors promot- ing quality of life have resulted in health issues in the elderly [1]. Aging is a natural phenomenon associated with reduced physical, psychological, and psychological activities. The physiological changes are similar in different people, although the speed of changes can be different. Lack of attention to body and mind during this period accelerates the aging process and ultimately leads to premature death [2]. This highlights the importance of proper planning in the aging period. An

\section{* Corresponding Author:}

Omid Jamshidi, PhD Student

Address: Department of Sports Management, Faculty of Physical Education and Sports Sciences, University of Mazandaran, Babolsar, Iran.

Tel: +98 (912) 8837768

E-mail: omidjamshidi88@yahoo.com 
ideal urban space is a space where citizenship justice is respected, and it can be used by all citizens, including vulnerable people [3]. In this regard, the present study was conducted with the aim of fitting optimal sports site selection criteria for the elderly.

\section{Methods \& Materials}

The descriptive-analytic research method was employed in this applied research work, which was approved by the Research Ethics Committee of Mazandaran University. This field study was conducted in three phases. In the first phase, six important criteria for optimal sports site selection of older adults were determined by studying library resources. The indices extracted by a structured interview tool were used by eight experts in the field of sports and sports facilities for pair-wise comparison.

The Expert Choice Software was used to analyze the data collected in this study. Since each type of sports facility has its own characteristics, they cannot be all together in the same place. In the second phase, 27 swimming pools of Karaj were selected, and the elderly referred to these sites were identified as the statistical population for this study. In this stage, a questionnaire designed using the indices determined in the first phase along with the four sub-criteria for each indicator was used. After collecting the data using the Analytical Hierarchy Process, the sub-criteria of each index were prioritized, and two sub-criteria with higher relative weights were determined for each of the indicators. Moreover, AutoCAD maps were prepared using AutoCAD 2015, Excel 2013, ArcGIS 9.3, Google Earth
7.1.8 or GPS device. In the third phase, we collected information for each of the prioritized sub-criteria to create a spatial research database. After exporting the layers used in the GIS environment and creating the topology, the information table was also completed and prepared for final analysis. Then, the location of each pool was determined according to the indicators.

\section{Results}

In the descriptive part of the research, the elderly are considered to be over 60 years old. According to the sampling method, 135 questionnaires were distributed, and finally, 127 completed questionnaires were collected. The majority of the sample size was male (66\%).

About $48 \%$ of the samples were aged between 60 and 65 years, $31 \%$ were aged between 65 and 70 years, and $21 \%$ were aged over 70 years. Regarding the employment situation, $41 \%$ of the samples were employed, and the remaining $59 \%$ were retired. Regarding the rate of sports activities per week, $46 \%$ of the respondents spent less than 6 hours a week while the remaining 54\% spent more than 6 hours a week on sports activities. According to the data obtained from the pair-wise comparison matrix, the most effective criteria are accessibility, closeness to other urban utilities, safety, density of sports facilities, topographical features, and privacy.

The three factors of accessibility, closeness to other urban utilities, and safety had the highest relative weights and were identified as important indicators. These factors, along with the sub-criteria for each criterion, were provided to some older adults attending sports facili-

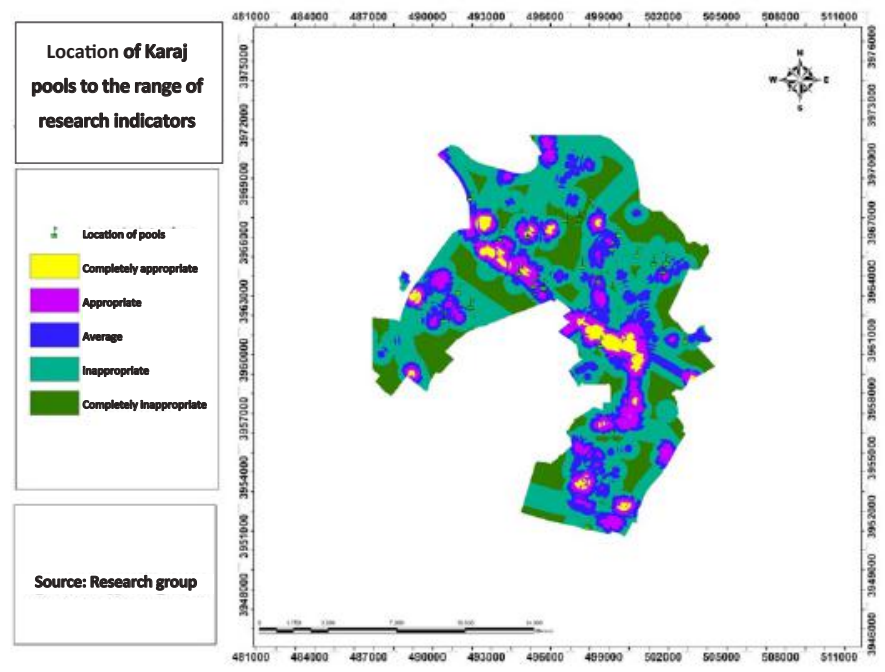

Figure 1. The location of the pools in Karaj concerning the range of indicators

IRANGAN Jounnalof 
ties. The results showed that among the sub-criteria, access to metro lines and highways, closeness to parks and green spaces as well as cultural centers and appropriate location relative to medical and fire-fighting centers had achieved higher relative weights. The maps of each sub-criterion were obtained from the relevant organizations, and the geographic locations of the pools in Karaj were determined (Figure 1). The areas with distance 0 to 200 meters, 200 to 400 meters, and 400 to 600 meters of the desired feature range were identified. Finally, the locations of different pools in Karaj were shown based on the research indicators in a five-item scale (completely appropriate, appropriate, medium, inappropriate, and completely inappropriate).

\section{Conclusion}

The findings of this study showed that the most important factors affecting the optimal sports site selection for the elderly are accessibility, closeness to other urban utilities, safety, density of sports facilities, topographical features, and privacy. This finding is consistent with the studies by Zohrevandian et al. (2015) [18] and Hosseini et al. (2013) [6]. Access to metro lines and highways was found to be an important factor in accessing sports pools in Karaj. These studies had limitations such as the unavailability of an integrated research sample, a survey of a group of sports facilities (pools), and the difference in the types of customers. Certainly, sports activity in the elderly, in addition to maintaining mobility and reducing diseases, brings about excitement and rejuvenation, resulting in a healthy life.

\section{Acknowledgments}

This research was extracted from the corresponding author's $\mathrm{PhD}$ dissertation in the Department of Sports Management, Faculty of Physical Education and Sports Sciences, University of Mazandaran, Babolsar, Iran.

\section{Conflict of Interest}

The authors declared no conflicts of interest. 


\title{
مناسبسازى شاخص هاى انتخاب مكان بهينه ورزشى سالمندان با استفاده از مدل تحليل سلسله

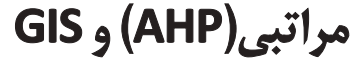

"اميد جمشيدى'، مرتضى دوستى باشا'، سيد محمدحسين رضوى'، محمود كودرزى'

ا - كروه مديريت ورزشى، دانشكده تربيت بدنى و علوم ورزشى، دانشكاه مازندران، بابلسر، ايران.

\begin{abstract}
בת

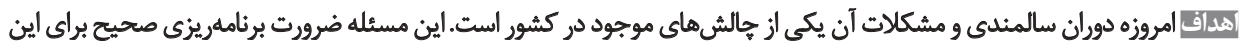

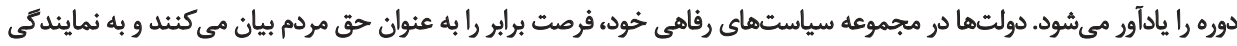

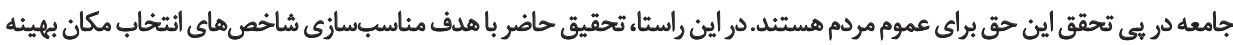

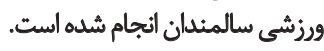

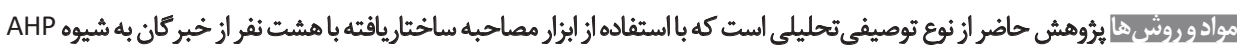

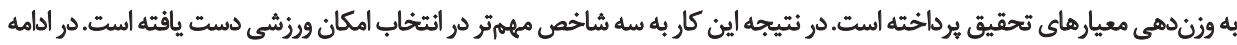

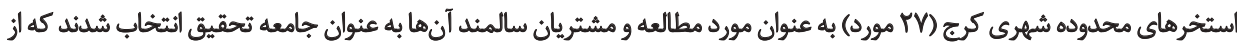

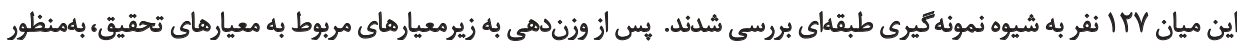

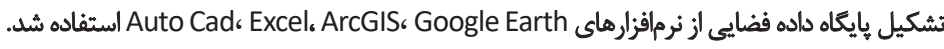

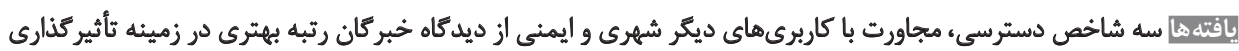

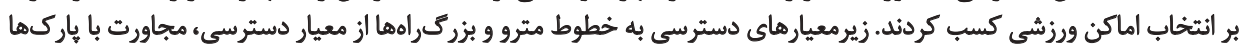

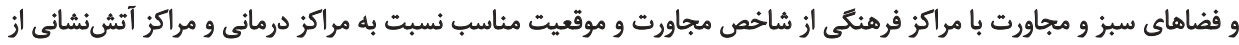

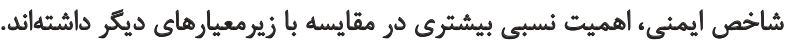

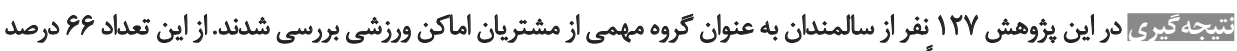

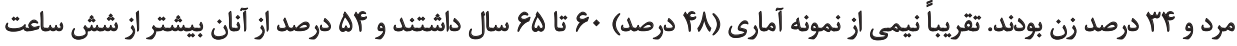

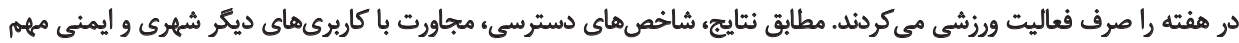

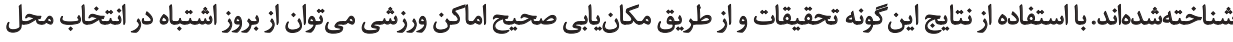

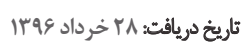

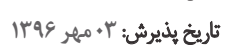

تعداد افراد سالمند در سراسر جهان شده است. در سالهاى

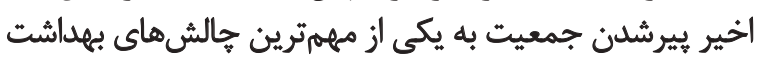

دوران سالمندى يك يديده طبيعى است كه در آن فعاليتهاى

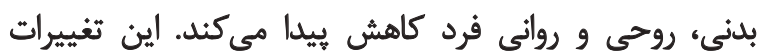

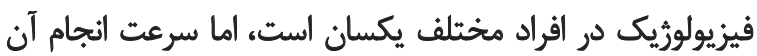

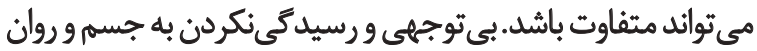

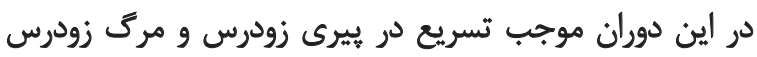

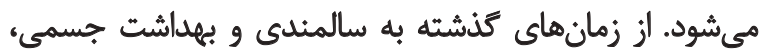

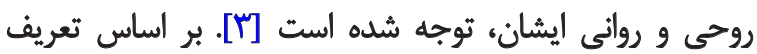

امروزه ورزش يكى از مهمترين، جذابترين و مؤثرترين رفتارها

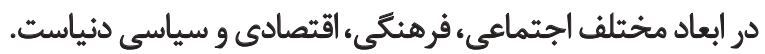

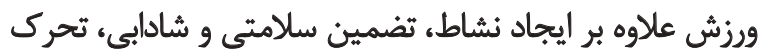

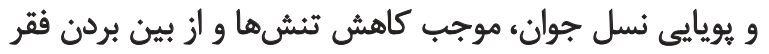

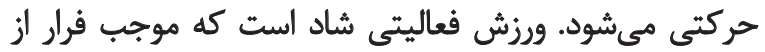

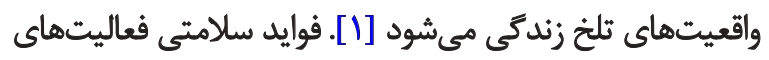

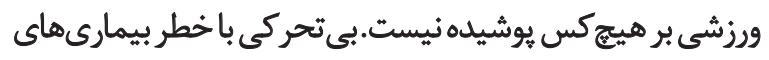

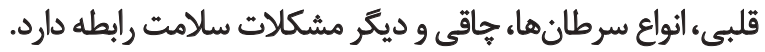

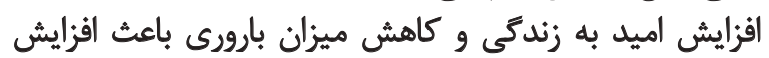




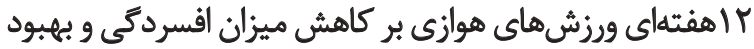

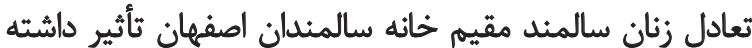

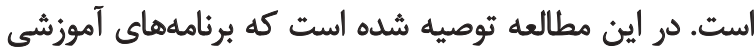

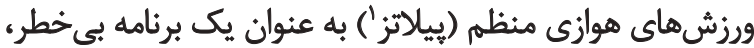

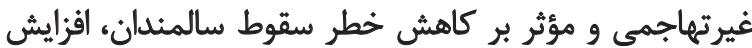

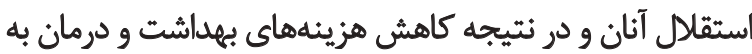

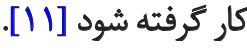

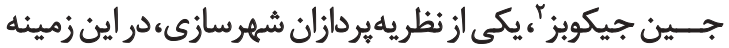

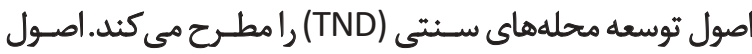

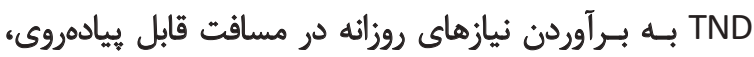

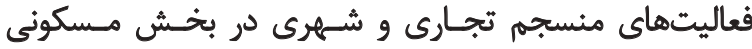

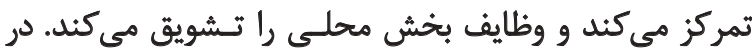

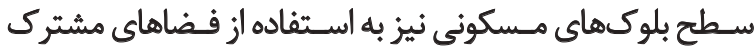

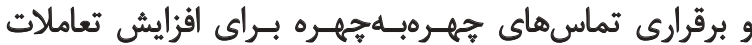

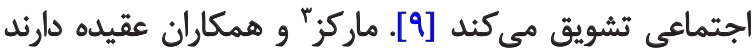

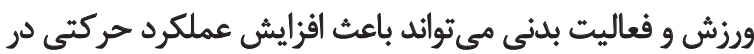

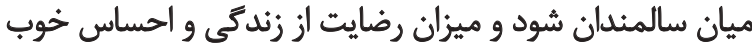

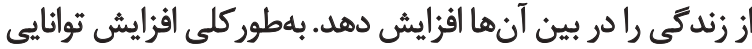

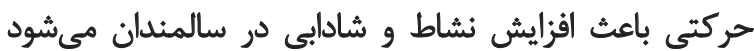

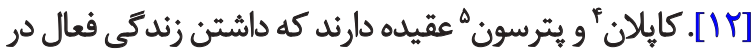

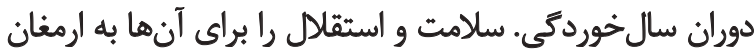

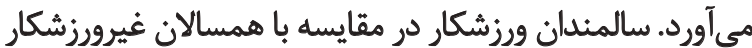

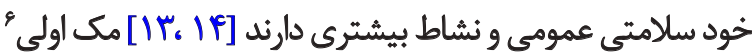

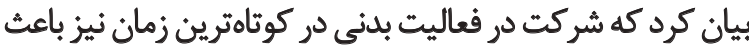

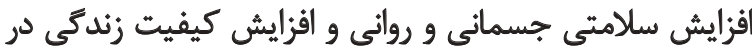

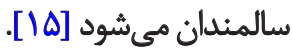

ضرورت برداختن بـهـ فــاهاى مناسـب بـراى سـالمندان در

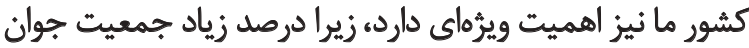

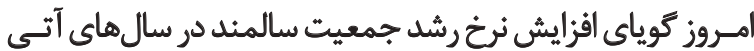

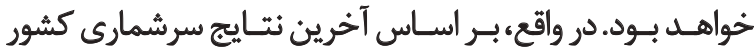

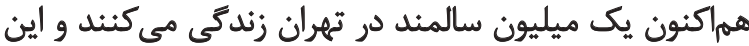

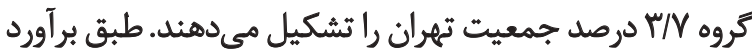

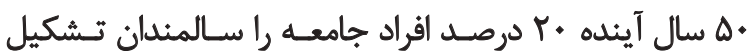

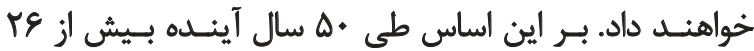

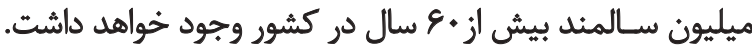

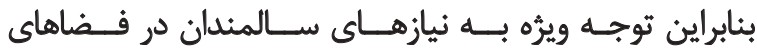

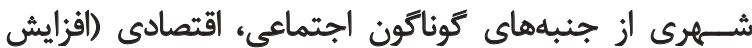

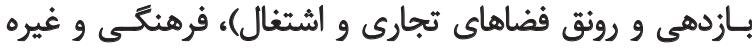

\section{Pilates}

2. Jane Jacobs

3. Marquez

4. kaplan

5. Patterson

6. Mcaule
سازمان جهاني بهداشت هر فرد بيش از ·و سال سالمند ثلقى

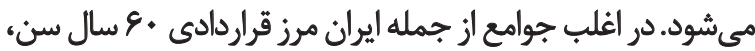

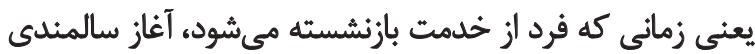

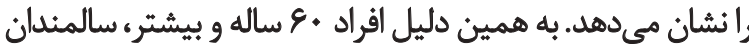

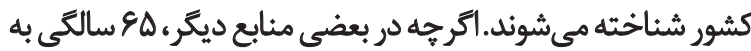

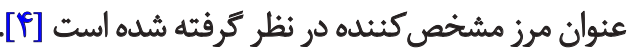

سالمندى دوران حساسى از زندكى است و توجه به مسائل و

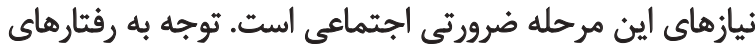

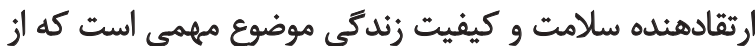

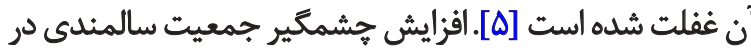

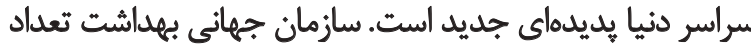

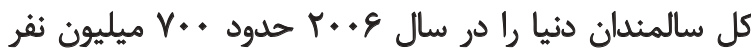

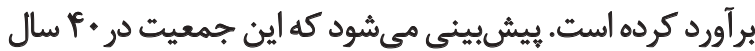

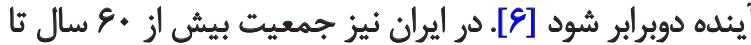

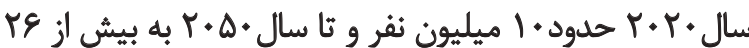

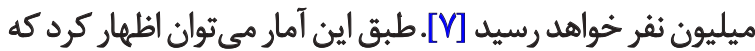

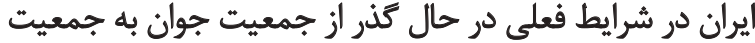

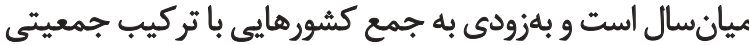

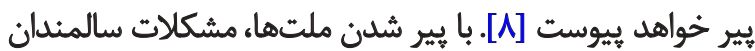

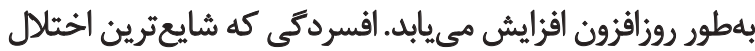

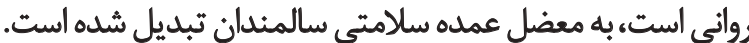
فضاي شهرى ايدهآل، فضايى است كه در آن عدالت شهروندى آندي

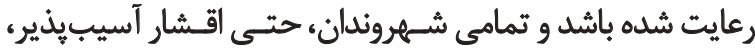

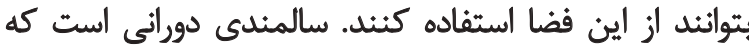

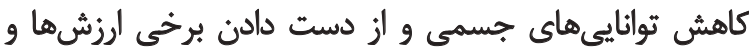

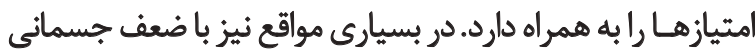

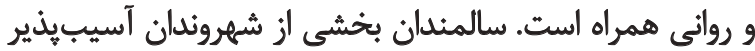

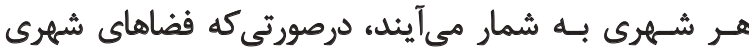

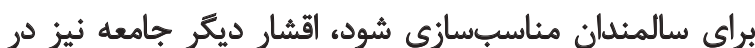

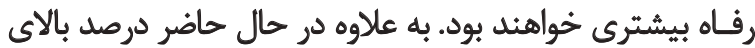

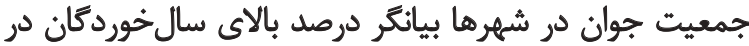

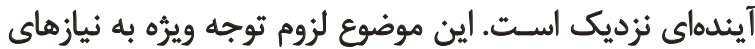

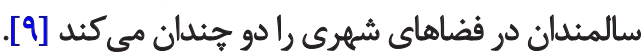
بهمنظور بهرهمندى سالمندان از زندگى شاد و مسرت آور و نيز

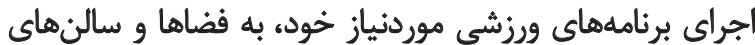

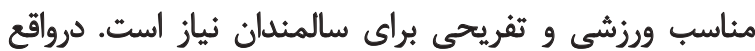

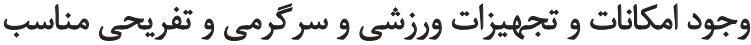

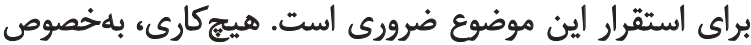

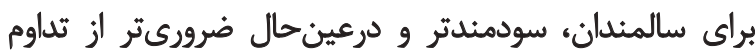

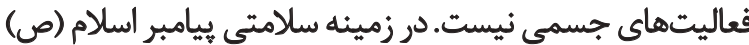

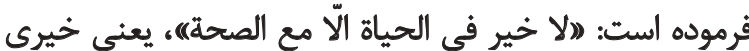

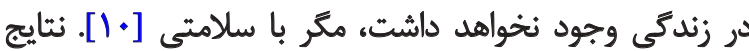

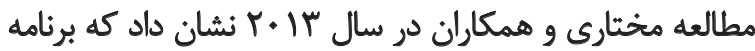




$$
r_{i j}=\frac{\overline{a_{\imath \jmath}}}{\sum_{i=1}^{n} \overline{a_{\imath \jmath}}}
$$

بعد از عادى كردن از مقادير هر سطر ميانكين موزون باضريب

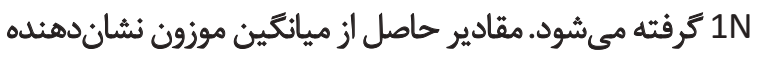

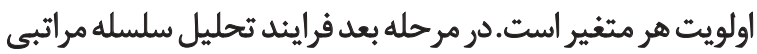

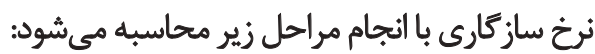

$$
\begin{aligned}
& I . I=\frac{\lambda_{\max }-n}{n-1} \\
& C . I=\frac{I . I}{R . I}
\end{aligned}
$$

در فرمول فوق R.I بيان كنتده مقدار شاخص تصادفي است و

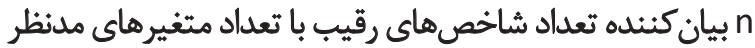

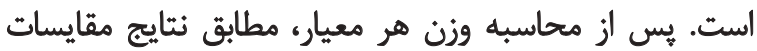

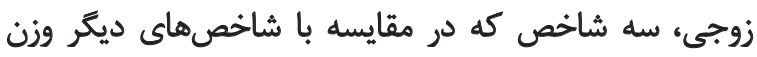

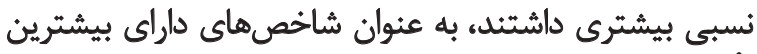

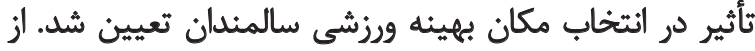

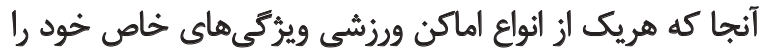

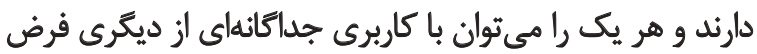

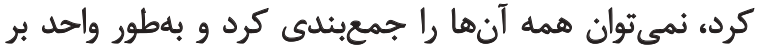

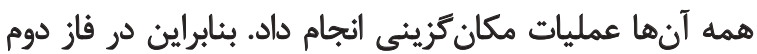

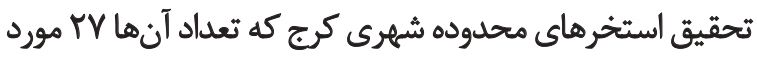

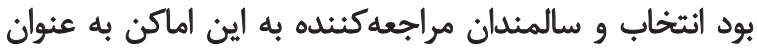

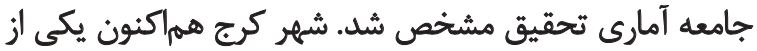

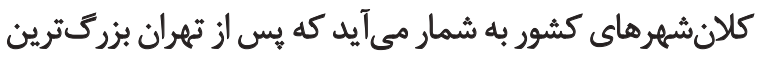

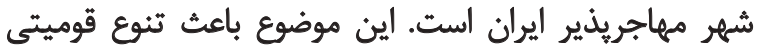

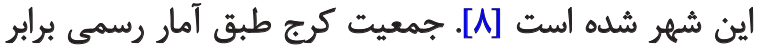

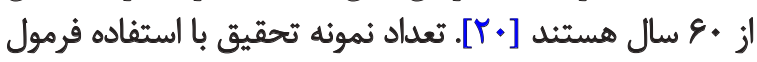

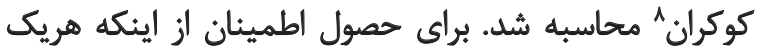

8. Cochran

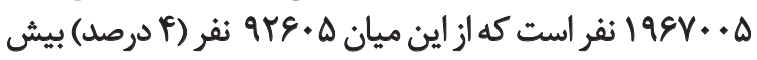

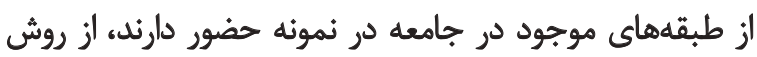

اهميت ويرُماي دارد [9].

با توجه به ايثكه ثاكنون تحقيقى در راستاى مناسبسازى

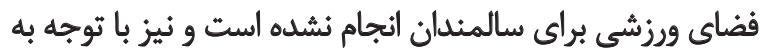

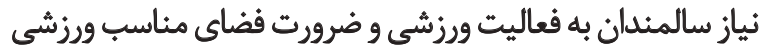

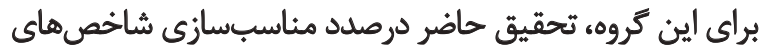

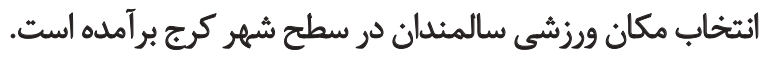

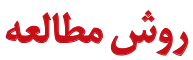

روشي تحقيق حاضر، توصيفي تحليلي و نوع آن كاربردى است

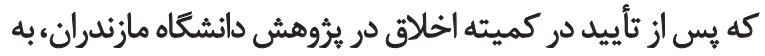

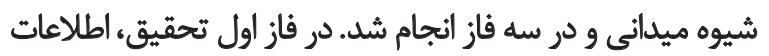

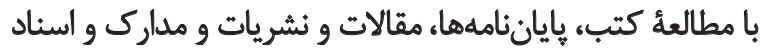

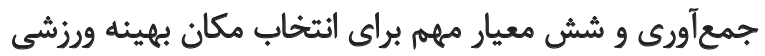

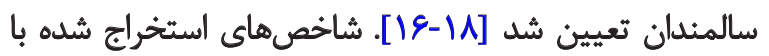

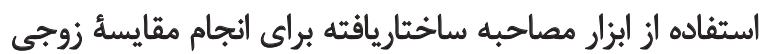

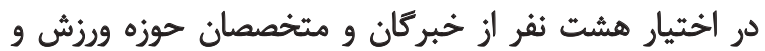

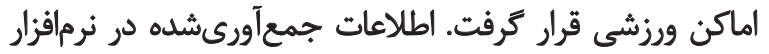

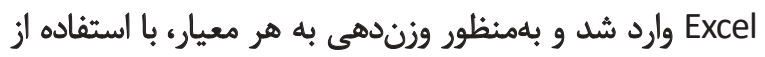

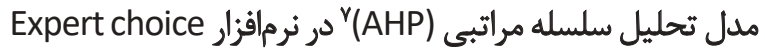

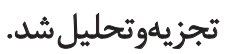

فرايئد تحليل سلسله مراتبي بر هايه تصميمزيرى بر اساس

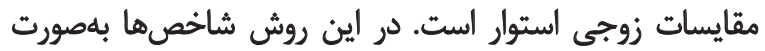

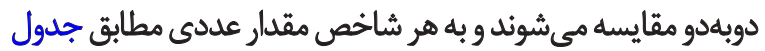

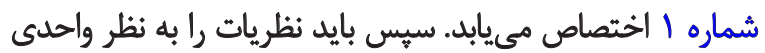

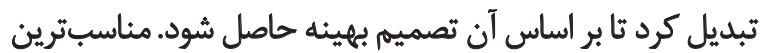

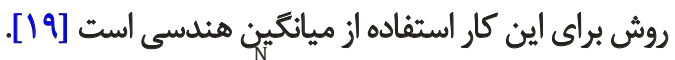
$\bar{a}_{i j}=\left(\prod_{\mathrm{K}=1}^{\mathrm{N}} \mathrm{a}_{i j}{ }^{(\mathrm{k})}\right)^{\frac{1}{\mathrm{~N}}}$

هي از آن براى تعيين اولويت از عمل عادىسازى استفاده

مي شود:

7. Analytic Hierarchy Process

جدول ا. مقايسه زوجى در تحليل سلسله مراتبى

\begin{tabular}{|c|c|}
\hline مقدار عددى & درجه الهميت در مقايسه زوجى \\
\hline 1 & الزخش برابر \\
\hline$r$ & ارزش اندكى بيشتر \\
\hline$a$ & ارزش بيشتر \\
\hline$\checkmark$ & ارزش خيلى بيشتر \\
\hline 9 & الرزش مطق \\
\hline r.fEA & الرزش بيئايين \\
\hline
\end{tabular}
مقدار عددى درجه الهميت در مقايسه زوجى 
يافتهها

در اين يُروهش در بخش توصيفى تحقيق منظور از سالمندان

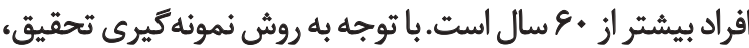

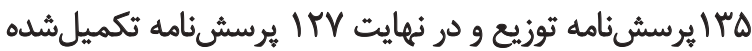

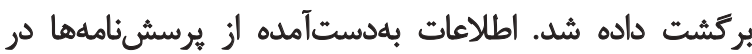
جدول شماره ب آورده شده است. همان كونه كه از جدول شماره r مشاهده مي مشود، بيشترين

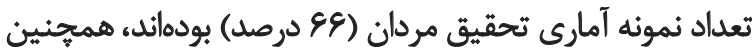

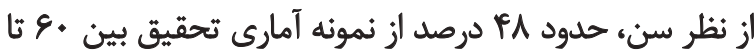

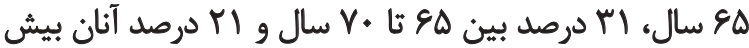

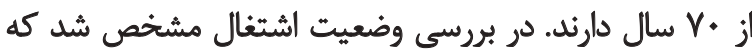

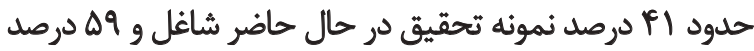

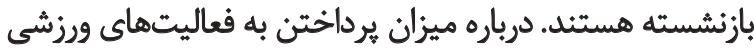

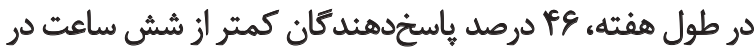

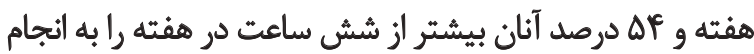
فعاليتهاى ورزشى اختصاص مى دهند.

اطلاعات مورد نياز براى مناسبسازى شاخصهاى انتخاب

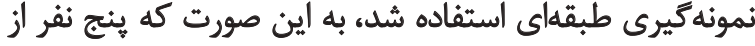

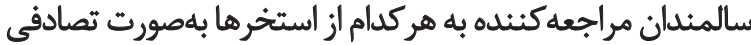

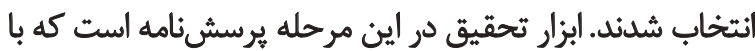

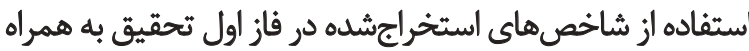
هجار زيرمعيار براى هر شاخص طراحى شد. يس از كردآورى اطلاعات با استفاده از مدل تحليل سلسله

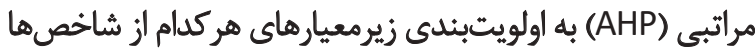

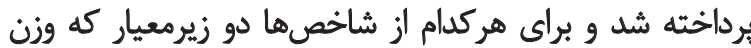

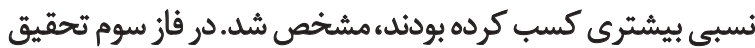

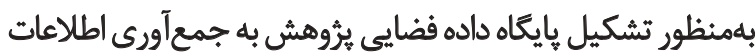

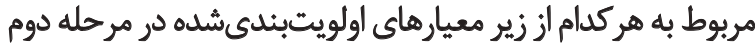

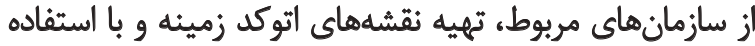

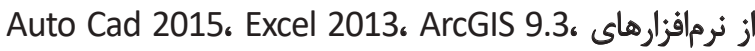

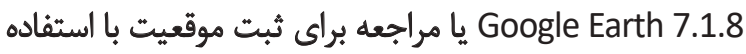

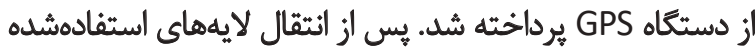

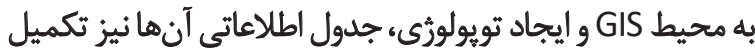

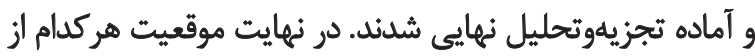

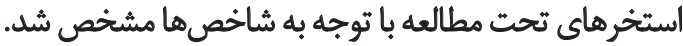

جدول r. ويثٔكى هاى جمعيتشناختى نمونه تحقيق

\begin{tabular}{|c|c|c|c|c|c|c|c|c|c|}
\hline \multicolumn{2}{|c|}{ فعاليت ورزشى در هفته } & \multicolumn{2}{|c|}{ وضعيت أشتغال } & \multicolumn{3}{|c|}{ سن } & \multicolumn{2}{|c|}{ جنسيت } & \multirow{2}{*}{ توصيفى } \\
\hline سيشيتر از 7 & كمتر ازئ 7 & بازْشُسته & شاغل & 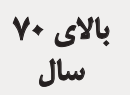 & عال & 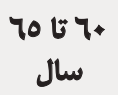 & j & هرد & \\
\hline 81 & $\Delta q$ & VQ & $\Delta r$ & $r V$ & rq & 81 & $R$ & Ap & تعلداد \\
\hline$\Delta F$ & is & $\Delta q$ & Fi & $M$ & r & iA & $m$ & 98 & فراوانى \% \\
\hline
\end{tabular}

جدول ץ. ماتريس مقايسات زوجى معيارهاى اصلى تحقيق

\begin{tabular}{|c|c|c|c|c|c|c|c|c|}
\hline & $F$ & $E$ & D & C & B & A & \multirow{2}{*}{ ماخصها } & \\
\hline شاخصص & مجاورت & دسترسى & رعايت حريم & تويوكرافى & تراكم & ايمنى & & \\
\hline.$/ 190$ & V/R & VEY & P/qY & $P / q 1$ & r/Nr & - & ايمنى & A \\
\hline $.1 \cdot n$ & $\mathbb{Q} / \mathbb{A}$ & $P / T P$ & INE & $1 / M$ & - &.$/ 48$ & تراكم & B \\
\hline$+1+49$ & $\Delta / \Delta$. & $8 / \ldots$ & $1 / \cdot r$ & - & - /Ar & $t / r$ & تويوكرافي & C \\
\hline $1+40$ & $\Delta / \cdot \varphi$ & $8 / 9 f$ & - &.$/ 9 V$ & $.1 \Delta \%$ & 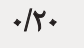 & رعايت حريم & D \\
\hline.$/ R A \Delta$ & $m / \cdot 1$ & - &.$/ 1 f$ &.$/ 18$ & 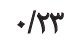 & .181 & دسترسي & $E$ \\
\hline - /TAF & - & ז"ז/. & .119 &.$/ M$ & .119 & .181 & مجاورت & $F$ \\
\hline
\end{tabular}


جدول F. ماتريس مثايسات زوجى شاخص دسترسى

\begin{tabular}{|c|c|c|c|c|c|c|}
\hline \multirow{2}{*}{ شاخص نسيى } & D & $C$ & B & $A$ & \multirow{2}{*}{\multicolumn{2}{|c|}{ شاخصير دعيار هائري سيى }} \\
\hline & راههاى فرعى & هترو & بزركراهذا & راههاى اصلى & & \\
\hline .1 .9 & retpr & 81ea & $\Delta / 1 \Lambda$ & - & راهذاي أصلى & A \\
\hline.$/ \mu$ & virq & T/Vq & - &.$/ 19$ & بزرئرها & B \\
\hline $.1 \Delta 8$ & V/ad & - & . $r \Delta$ &.$/ F$ & مثرو & C \\
\hline .1 .4 & - &.$/ 1 T$ & rות &.$/ r q$ & راههاى فرعي & D \\
\hline
\end{tabular}

دارند، به عنوان شاخصهاى مههمتر مشخص شدند. در فاز دوم تحقيق سه عامل كه از ديدگاه خبركان رتبه

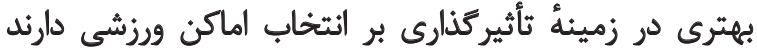

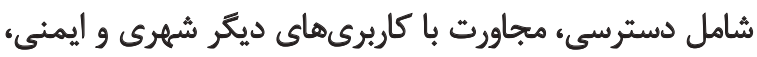

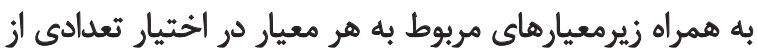

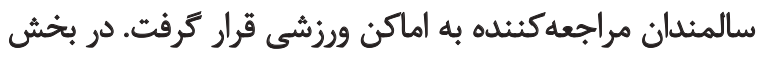

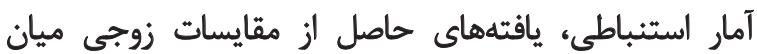

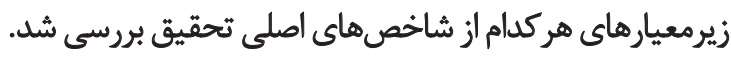

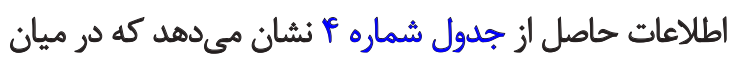

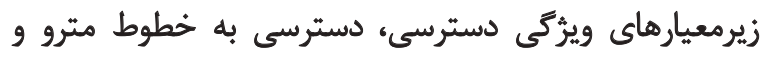

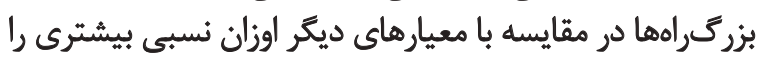

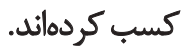

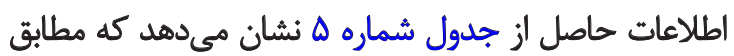

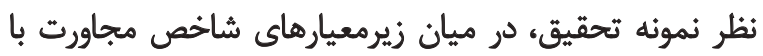

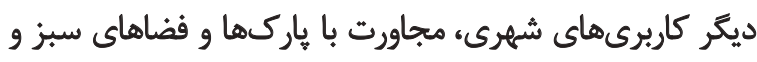

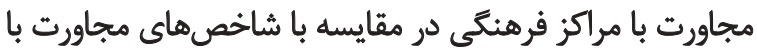

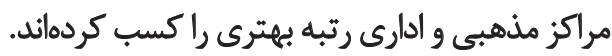

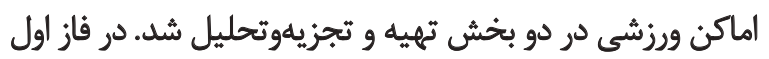

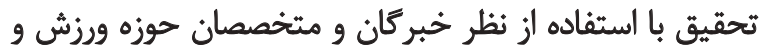

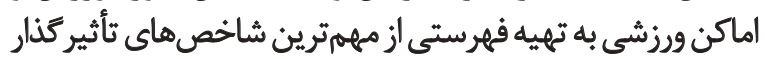

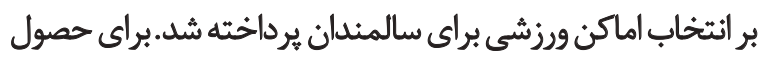

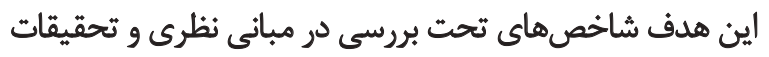

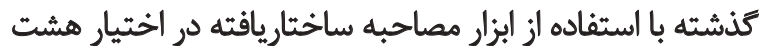

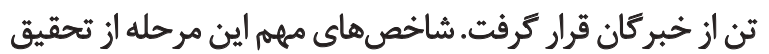

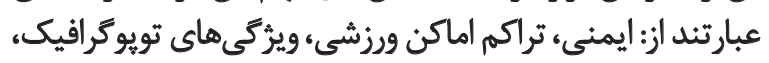

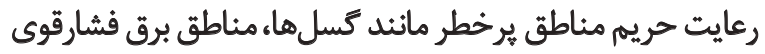
و غيره، دسترسى و مجاورت با كاربرى هانى ديكر شهرى با استفاده از روش تحليل سلسله مراتبى، معيارهاى مدنظر

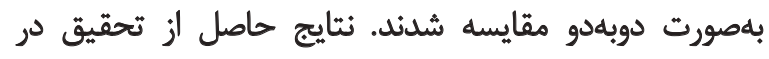

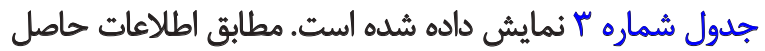

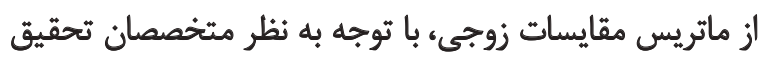

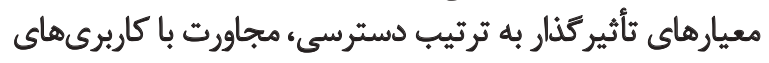

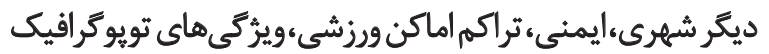

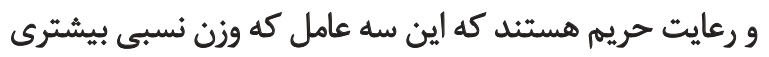

جدول ه. ماتريس مقايسات زوجى شاخص مجاورت

\begin{tabular}{|c|c|c|c|c|c|c|}
\hline \multirow[b]{2}{*}{ وزأنمب نسبى } & D & C & B & A & \multirow[b]{2}{*}{ 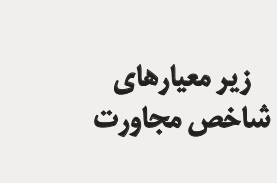 } & \\
\hline & مراكز ادارى & مراكز مذهبي & مراكز فرهنكى & ساركها و فضاهاي & & \\
\hline $.10 A$ & g/Te & SlAT & r/AT & $=$ & باركها و فضاهاي سبز & A \\
\hline.$/ M A$ & P/A9 & $\Delta / q C$ & - &.$/ M A$ & مراكز فرهنكى & B \\
\hline .1 .8 & $1 / 48$ & - & .118 &.$/ 14$ & مراكز هذهبي & C \\
\hline .1 .8 & - &.$/ M 9$ &.$\pi$ &.$/ 10$ & هراكز ادارى & D \\
\hline
\end{tabular}


جدول \& ماتريس مقايسات زوجى شاخص ايمنى

\begin{tabular}{|c|c|c|c|c|c|c|}
\hline \multirow{2}{*}{ شأن نُشيى } & D & C & B & A & \multirow{2}{*}{ ياخص معيار ايمنى } & \\
\hline & مراكز مديريت & مراكز بليس & مراكز درمانى & مراكز أتشنشانى & & \\
\hline . & P/OV & $r / r^{m}$ & r/q & - & مراكز آتش نشاني & A \\
\hline.$|8|$ & elve & sle. & - &.$/ T \Delta$ & مراكز درماني & B \\
\hline .11 & T/AF & - &.$/ 10$ & $/ r$ & مراكز بليس & C \\
\hline$+1 . \infty$ & - & . $/ 4 \Delta$ &.$/ 1 P$ &. $\mid M$ & مراكز مديريت بحران & D \\
\hline
\end{tabular}

استخرهاى مختلف شهر كرج را نسبت به شاخص هاى تحقيق در

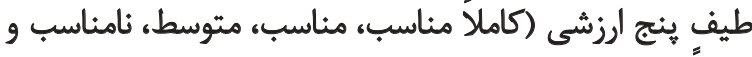
كاملاً نامناسب) نمايان ساخت (جدول شماره (1).

ث

سالمندان بخشى از شهروندان آسيب ئذير بهحساب مى آيند كه

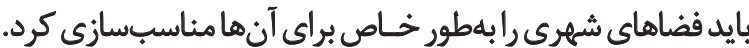

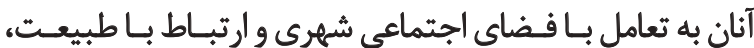

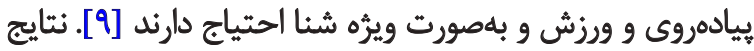

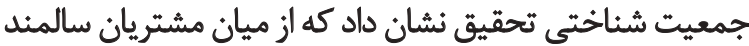

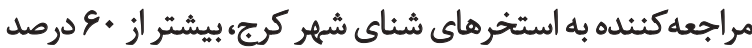

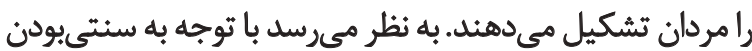

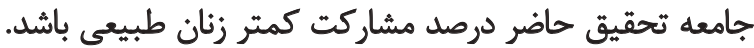

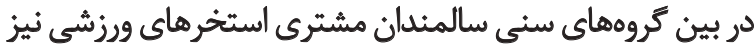

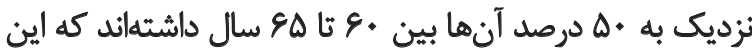

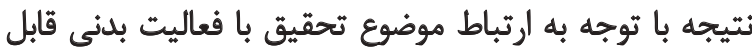

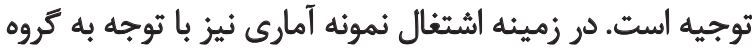

اطلاعات حاصل از جدول شماره \& نشان ميدهد در ميان

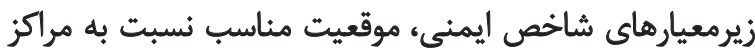

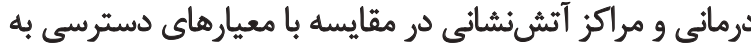

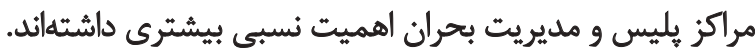

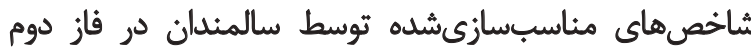

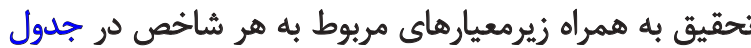

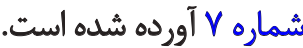
در ادامه يُروهش نقشههاي مربوط به هر زير معيار از سازمان هاي

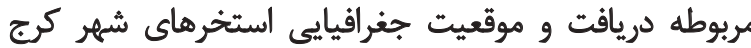

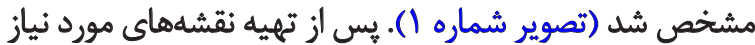

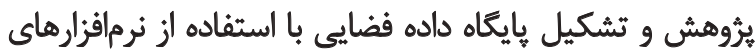
Auto Cad، Excel، ArcGIS، Google Earth براى ثبت موقعيت با استفاده از دستكاه GPS و مشخصشدن

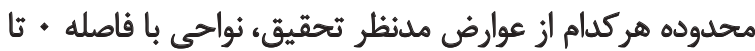

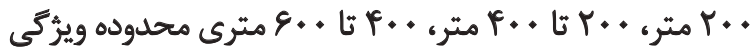

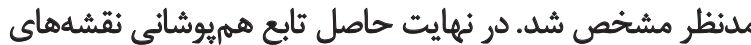

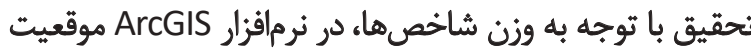

جدول V. شاخصهاى مثاسبسازي شده به همراه زير معيارها

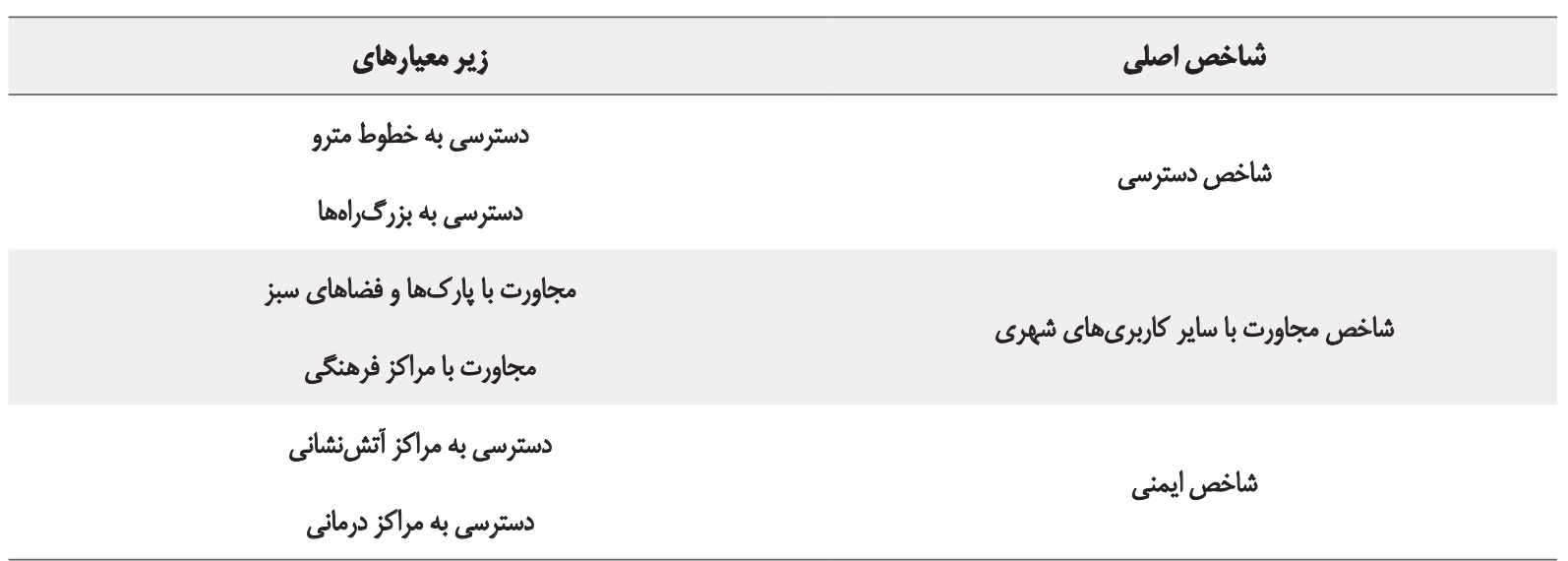




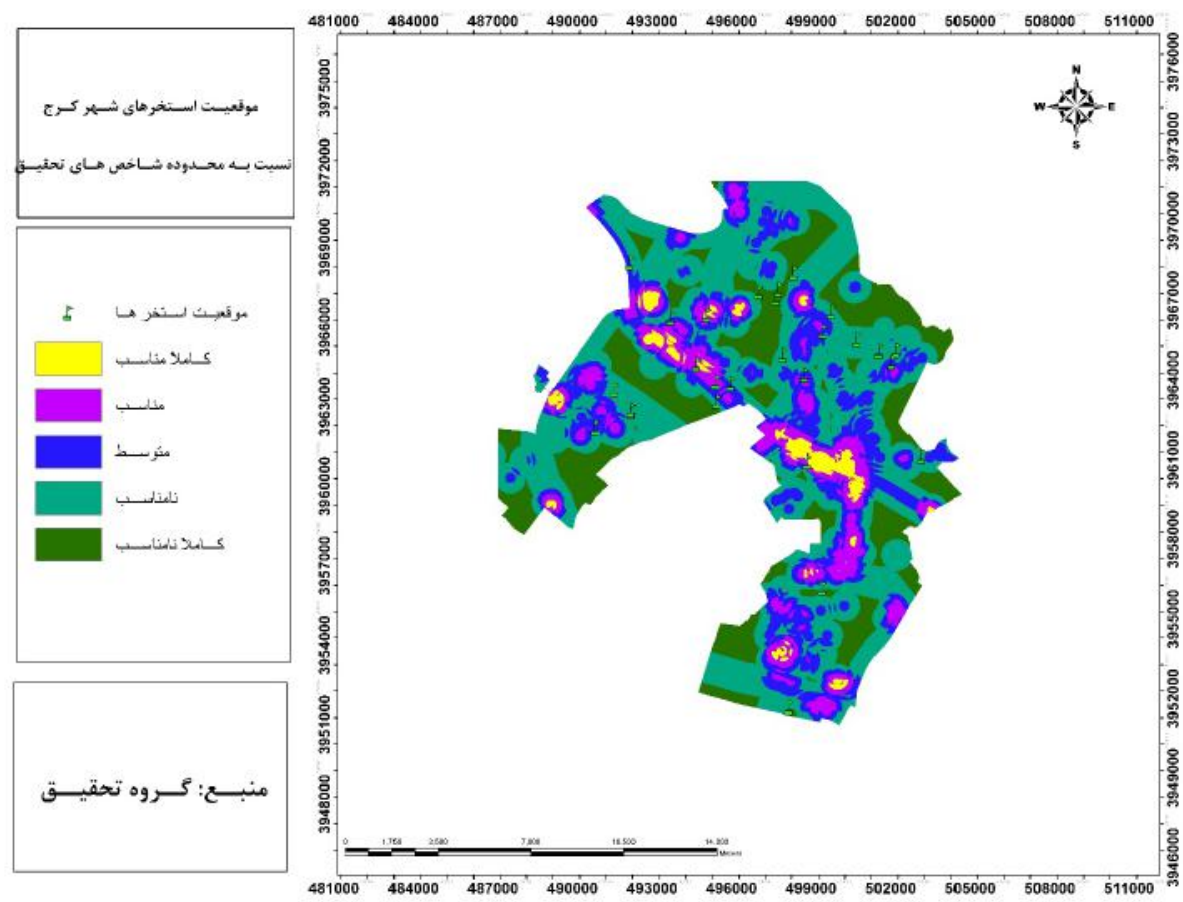

il

تصوير ا. موقعيث استخرهاي شهر كرج نسبت به محدوده شاخصها

جدول ^. وضعيت استخرهاى شهر كرج نسبت به شاخصهاى تحقيق

\begin{tabular}{|c|c|c|c|c|c|}
\hline وضعيت دسترسى & نام استّحر & رديف & وضعيت دسترسى & نام استخرّ & رديف \\
\hline متوسط & دانش & 10 & كاملاً هناسب & مهتاب & 1 \\
\hline نامناسب & اسيارس & 18 & نامناسب & مشكات & $r$ \\
\hline متوسط & بهشت شرق & iv & ن نامناسب & ترمه & $r$ \\
\hline نامناسب & i & $M$ & كاملأ نامناسب & امير مرشدل & r \\
\hline نامناسب & نشاط & 19 & كاملأ نامناسب & ساويز & $\Delta$ \\
\hline نامناسب & همايون & $r$. & مناسب & آميتيس البرز & 8 \\
\hline متونط & حَوارزمي & M & كاملأ نامناسب & آيدين & $\checkmark$ \\
\hline ثامناسب & يامجال & r & مناسب & آفتاب البرز & $\Lambda$ \\
\hline نامناسب & يادآوران & r & متوسط & ارمغان تربيت & 9 \\
\hline نامناسب & شهباى فرديس & rf & نامناسب & انرُّى البرز & 1. \\
\hline متوسط & ايثار & ro & نامناسب & سوم خرداد & 11 \\
\hline كاملاً مناسب & شريعتى & re & متوسط & مفتح & ir \\
\hline \multirow[t]{2}{*}{ ن امناسب } & آزادكان & $r$ & متوسط & السيالين & ir \\
\hline & & & نامناسب & حس ثششم & if \\
\hline
\end{tabular}




\section{نتيجهئيرىنهايي}

از طريق مكانيابى مناسب اماكن ورزشى مىثوان از بران بروز

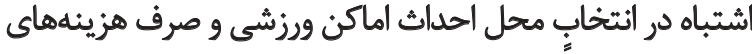

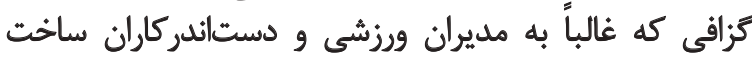

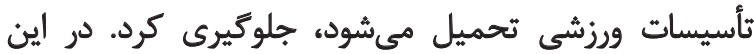

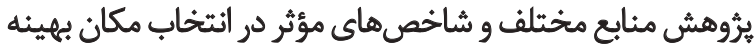

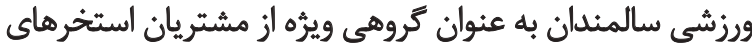

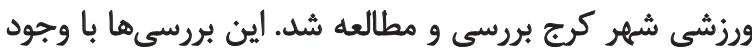

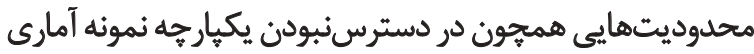

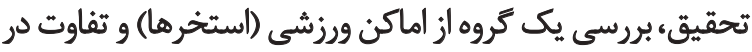
نوع مشتريان اين اماكن صورت يذيرفت إنى

بىترديد بخشى از نيروهاى فعال در جامعه افراد سالمند

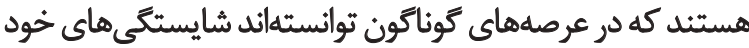

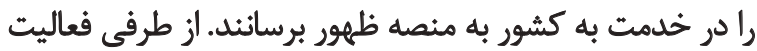

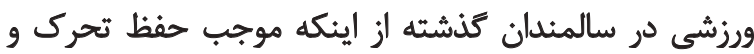

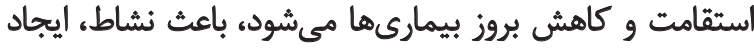

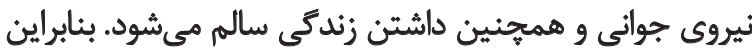

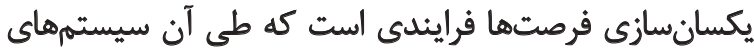

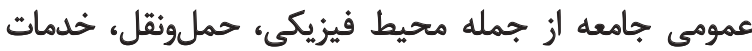

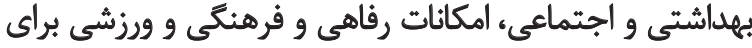

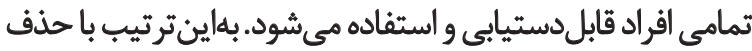

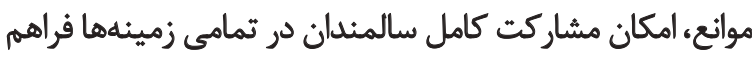

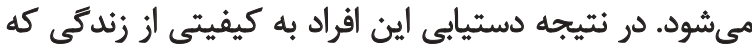

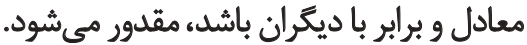

$$
\text { تشكر و قدردافي }
$$

يروهش حاضر بركرفته از باياننامه دكترى نويسنده مسئول

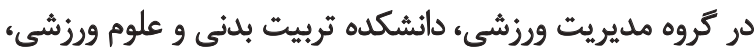

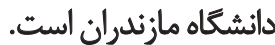

مدنظر، حدود •و درصد افراد بازنشسته و بقيه شاغل هستئد.

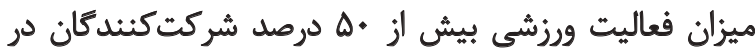

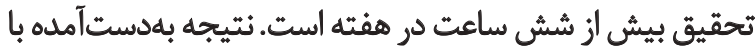

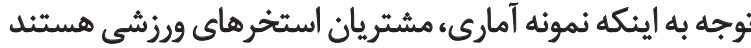
و اين خود دليلى بر تمايل اين كروه به فعاليت ورزشي است،

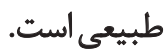

در ادامه، يافتههاى اين تحقيق نشـان داد كه مطابق با نظر

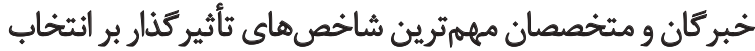

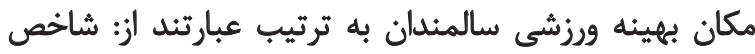

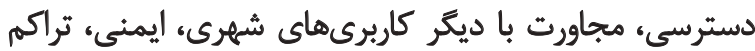

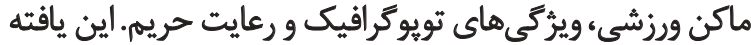

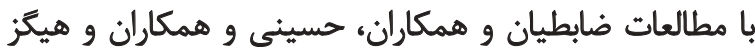

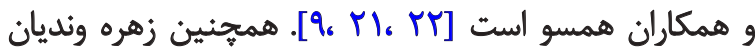

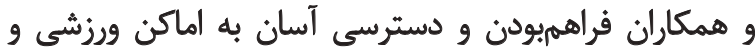

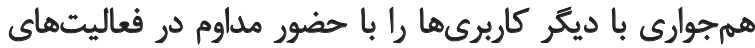

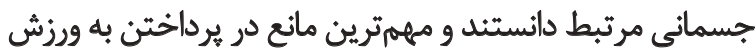

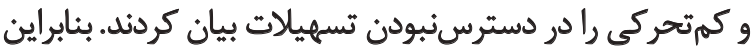

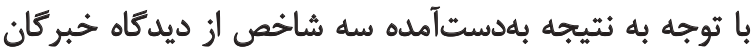

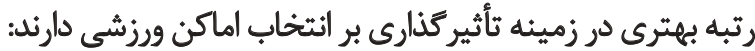

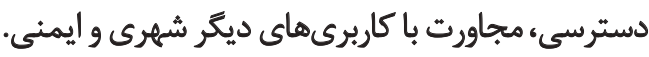
نتايج مقايسات زوجى زيرمعيارهاى شاخص دسترسى دمري

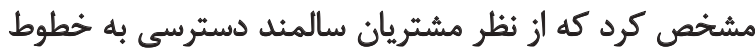

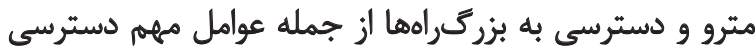

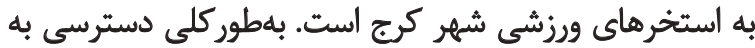

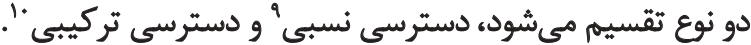

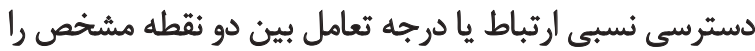

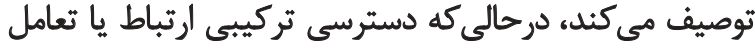

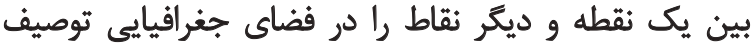

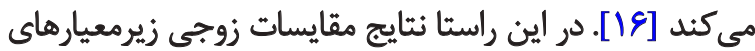

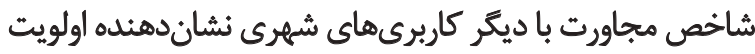

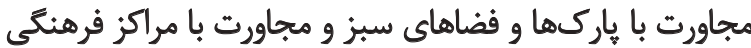

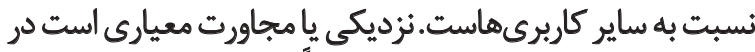

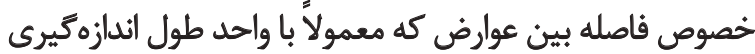

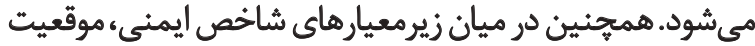

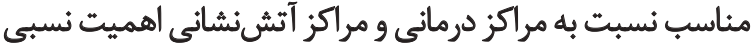

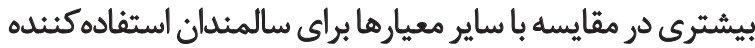

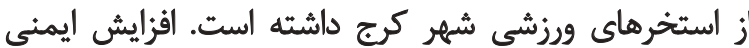

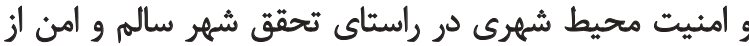

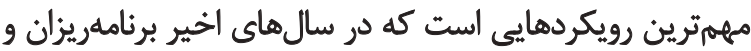

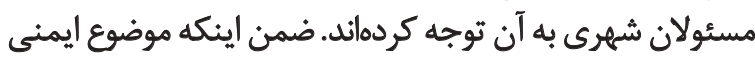

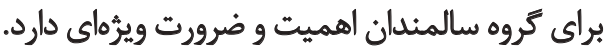




\section{References}

[1] Alipour F, Sajadi H, Forouzan A, Biglarian A, Jalilian A. [Elderly quality of life in Tehran's district two (Persian)]. Iranian journal of ageing. 2008; 3(3-4):75-83.

[2] Ebrahami K. [Sports and dialogue among civilizations (Persian)]. Paper presented at: The First Congress of Women's in Physical Education Organization. 5 August 2002; Tehran, Iran.

[3] Gholizadeh A, Shirani E. [The relation between personal, family, social and economic factors with the rate of life satisfaction of aged people of Isfahan (Persian)]. Applied Sociology. 2010; 21(37):69-82.

[4] Hamedivafa, F. [Ibn Sina and Jorjani's view on elderly health (Persian)]. Teb va Tazkiyeh. 2008; 16(1-2):70-7.

[5] Higgs G. Integrating multi-criteria techniques with geographical information systems in waste facility location to enhance public participation. Waste Management \& Research. 2006; 24(2):105-17. doi: $10.1177 / 0734242 \times 06063817$

[6] Hoseini SS, Kashef SM, Seyedameri MH. [Locating sport gyms through geographical information system; Case study: Saghez city (Persian)]. Pazhooheshhay-e Karbordi dar Modiriyat-e Varzeshi. 2012; 2(5):25-34.

[7] Sadock BJ, Sadock VA. kaplan and sadock's synopsis of psychiatry: behavioral sciences/clinical psychiatry. Philadelphia, Pennsylvania: Lippincott Williams \& Wilkins; 2007.

[8] Wikipedia. [Karaj (Persian)] [Internet]. 2017 [Updated 2017 October 16]. Available from: https://fa.wikipedia.org/ wiki/\%DA\%A9\%D8\%B1\%D8\%AC

[9] Khalili M, Mohjelshoja M, Esmaeili S, Bateni, GH. [The role of exercise in the elderly from the perspective of Razi, Hakim moyasari, Ibn Sina and Jorjani (Persian)]. History of Medical Journal. 2011;3(7):57-76.

[10] Kochaki G, Hojjati H, Sanagoo A. [The Relationship between Loneliness and Life Satisfaction of the Elderly in Gorgan and Gonbad Cities (Persian)]. Journal of Research Development in Nursing \& Midwifery. 2012; 9(1): 61-68.

[11] Kwok RCW, Yeh AGO. The use of modal accessibility gap as an indicator for sustainable transport development. Environment and Planning A. 2004; 36(5):921-36. doi: 10.1068/a3673

[12] Garatachea N, Molinero O, Martínez-García R, Jiménez-Jiménez R, González-Gallego J, Márquez S. Feelings of well being in elderly people: Relationship to physical activity and physical function. Archives of Gerontology and Geriatrics. 2009; 48(3):306-12. doi: 10.1016/j.archger.2008.02.010

[13] Elavsky S, McAuley E, Motl RW, Konopack JF, Marquez DX, $\mathrm{Hu}$ L, et al. Physical activity enhances long-term quality of life in older adults: Efficacy, esteem, and affective influences. Annals of Behavioral Medicine. 2005; 30(2):138-45. doi: 10.1207/ s15324796abm3002 6

[14] Mokhtari M, Nezakatalhossaini M, Esfarjani F. The effect of 12week pilates exercises on depression and balance associated with falling in the elderly. Procedia - Social and Behavioral Sciences. 2013; 70:1714-23. doi: 10.1016/j.sbspro.2013.01.246

[15] Moti haghshenas, N. [Sociological aspects of aging populations and active aging challenges in Iran (Persian)]. Sociological Studies of Iran. 2011; 1(2):133-147.
[16] Salami M. [Presentation and comparison three models of AHPTAXONOMY, FAHP-FTOPSIS, FAHP-GCA in GIS space for site selection of sport places (Persian)] [PhD thesis]. Tehran: University of Tehran; 2014.

[17] Soltan Hoseini M, Alidoust Ghahfarokhi E, Farahani A. [ Traffic and Natural Environmental Effects of Sport Stadiums On Urban Environment of Yazd (Persian)]. Journal of Sport Management Review. 2015, 6(24):15-30.

[18] Zohrevandian K, Asadi H, Ebrahimi F, Samadi M. [Determine and prioritizing criteria for site selection of sport facilities for equality in access using Analytical Hierarchy Process (AHP) (Persian)]. Sport Management. 2015; 29:795-814.

[19] Shakeri A, Salami F. [Factors affecting investment attraction in Chabahar Free zone and prioritizing them using AHP mathematical technique (Persian)]. Economics Research. 2006; 6(20):95-130.

[20] Statistical Center of Iran. [National Portal of Statistics (Persian)] [Internet] 2017 [Updated 2017 October 31, 2017]. Available from: https://www.amar.org.ir

[21] Nafisi A. [Research on the principles of traditional medical beliefs of the Iranian people (Persian)]. Esfahan: Mash'al; 1985.

[22] Patterson SL. Effect of treadmill exercise training on spatial and temporal gait parameters in subjects with chronic stroke: A preliminary report. The Journal of Rehabilitation Research and Development. 2008; 45(2):221-8. doi: 10.1682/jrrd.2007.02.0024 